\title{
High resolution studies of Broad Absorption Line radio-loud quasars
}

\author{
Marcin P. Gawroński* \\ Toruń Centre for Astronomy, N. Copernicus University, Gagarina 11, 87-100 Toruń, Poland \\ E-mail: motylekeastro.uni.torun.pl

\section{Magdalena Kunert-Bajraszewska} \\ Toruń Centre for Astronomy, N. Copernicus University, Gagarina 11, 87-100 Toruń, Poland \\ E-mail: magdaeastro.uni.torun.pl
}

\begin{abstract}
Broad absorption lines (BALs), seen in a small fraction of both the radio-quiet and radio-loud quasar populations, are probably caused by the outflow of gas with high velocities and are part of the accretion process. The presence of BALs is the geometrical effect and/or connected with the quasar evolution. Using the final release of the FIRST survey combined with a A Catalog of BAL QSOs (SDSS/DR3), we have constructed a new sample of compact radio-loud BAL QSOs, which included the majority of radio-loud BAL QSOs. The main goal of this project is to study the origin of BALs by analysis of the BAL QSOs radio morphology, their orientation and jets evolution, using EVN at $1.6 \mathrm{GHz}$ and VLBA at 5 and $8.4 \mathrm{GHz}$. We will discuss also the first multi-frequency radio observations of one very compact BAL quasar 1045+352 made using the VLBA, EVN, MERLIN and CHANDRA. We speculate that the most probable interpretation of the observed radio structure of $1045+352$ is the ongoing process of the jet precession due to internal instabilities within the flow.
\end{abstract}

10th European VLBI Network Symposium and EVN Users Meeting: VLBI and the new generation of radio arrays

September 20-24, 2010

Manchester Uk

\footnotetext{
* Speaker.
} 


\section{Introduction}

Broad absorption lines (BALs) - high ionization resonant lines (C IV 1549) and low ionization lines ( $\mathrm{Mg}$ II 2800), are seen in a small fraction of both the radio-quiet and radio-loud quasar populations (10-30\%, depends on selection criteria, e.g [[]]) . It is suggested that the presence of BALs is a pure geometrical effect [3] or/and is connected with the quasar evolution scheme [1].

BALQSOs are identified by means of their Balnicity Index $B I>0$ [13] but very recently a less restrictive criterion, Absorption Index $A I>0$ [12], has been defined and used. $B I$ is computed considering any absorption trough spanning $\geq 2000 \mathrm{~km} \mathrm{~s}^{-1}$ in width, absorbing at least $10 \%$ of the local continuum, and blueshifted by $\geq 3000 \mathrm{~km} \mathrm{~s}^{-1}$ with respect to the corresponding emission lines. The Absorption Index is computed in the same way as $B I$, but relaxing the $3000 \mathrm{~km} \mathrm{~s}^{-1}$ blueshift criterion and considering all absorption troughs with a blueshift $>0 \mathrm{~km} \mathrm{~s}^{-1}$ with respect to the corresponding emision lines, and with a width of at least $1000 \mathrm{~km} \mathrm{~s}^{-1}$. The validity of the latter definition has been recently questioned (e.g. [2] ]). The BALQSOs are classified into three subclasses: High-ionization BALs (HiBALs) contain strong, broad absorption high-ionization lines and are typicaly identified through the presence of CIV absorption; Low-ionization BALs (LoBALs) contain HiBAL features but also have absorption from low-ionization lines (MgII) present in optical spectra; FeLoBALs are LoBALs with exited-state FeII and FeIII absorption.

Theoretical models ([3], [10]) suggest that BALs are seen at high inclination angles, which means that the outflows from accretion disks are present near an equatorial plane. However, some recent numerical work indicates that it is also plausible to launch bipolar outflows from the inner regions of a thin disk (e.g. [11]). There is a growing observational evidence indicating the existence of polar BAL outflows (e.g. [16]). This means that there is no one simple orientation model which can explain all the features observed in BAL quasars.

The radio morphologies of radio-loud BAL quasars provide important additional information about their orientation and the direction of the outflow. Currently, there are only 17 compact radio-loud BAL quasars observed with VLBI in the literature (e.g. [河], [9]). About half of them have still unresolved radio structures even in the high resolution observations, the others have core-jet structures indicating some re-orientation or very complex morphology, suggesting a strong interaction with the surrounding medium [7]. Recent observations support the evolutionary scheme [8].

\section{Observations of BAL quasars}

\subsection{BAL quasar 1045+352 - summary}

$1045+352$ is a compact radio-loud CSS source. The spectral observations [15] have shown $1045+352$ to be a quasar with a redshift of $z=1.604$. It has also been classified as a HiBALQSO based upon the observed very broad C IV absorption, and it is a very luminous submillimetre object with detections at both $850 \mu \mathrm{m}$ and $450 \mu \mathrm{m}$ [15]. The radio luminosity of $1045+352$ at $1.4 \mathrm{GHz}$ is high $\left(\log L_{1.4 \mathrm{GHz}}=28.25 \mathrm{~W} \mathrm{~Hz}^{-1}\right)$, making this source one of the most radio-luminous BAL quasars. The Chandra X-ray observation of $1045+352$ [6] show that the X-ray emission of $1045+352$ is very weak in comparison to the other radio-loud BAL quasars which, together with the high value of the optical-X-ray index $\alpha_{o x}=1.88$, suggests a presence of an X-ray absorber close/in the BLR 


\begin{tabular}{c|c|c|c|c|c|c}
\hline $\begin{array}{c}\text { Source } \\
(1)\end{array}$ & $\mathrm{z}$ & $\begin{array}{c}\text { Type } \\
(2)\end{array}$ & $\begin{array}{c}\text { Absorption } \\
\text { index /AI/ } \\
(4)\end{array}$ & $\begin{array}{c}F_{1.4 \mathrm{GHz}} \\
/ \mathrm{mJy} / \\
(5)\end{array}$ & $\begin{array}{c}\alpha_{4.8}^{1.4} \\
(6)\end{array}$ & $\begin{array}{c}\text { Radio } \\
\text { morphology } \\
(7)\end{array}$ \\
\hline $0214-011$ & 2.462 & HiBAL & 791 & 218.0 & 0.60 & core-jet \\
$0753+373^{*}$ & 2.514 & HiBAL & 841 & 247.3 & 0.04 & core-jet \\
$0756+406^{*}$ & 2.021 & HiBAL & 1114 & 199.6 & 0.87 & core-jet \\
$0812+332^{*}$ & 2.426 & nHiBAL & 747 & 342.5 & 0.94 & core-jet/double \\
$0925+450 A^{*}$ & 1.904 & nHiBAL & 293 & 162.2 & -0.35 & core-jet? \\
$1002+483^{*}$ & 2.372 & HiBAL & 783 & 209.3 & 0.89 & core-jet \\
$1010+495^{*}$ & 2.201 & nHiBAL & 361 & 269.4 & 0.39 & core-jet/double \\
$1015+057$ & 1.938 & HiBAL & 441 & 296.6 & -0.09 & core-jet? \\
$1040+080$ & 2.665 & HiBAL & 1011 & 381.6 & 0.71 & double \\
$1054+036$ & 2.832 & HiBAL & 440 & 157.4 & 0.66 & core-jet \\
J1103+0232 & 2.514 & HiBAL & 460 & 166.0 & 0.34 & core-jet? \\
$1157+014$ & 2.000 & HiBAL & 2887 & 268.5 & 0.64 & unresolved \\
$1221+509^{*}$ & 3.488 & HiBAL & 413 & 228.8 & 0.59 & core-jet \\
$1403+411^{*}$ & 1.993 & HiBAL & 780 & 214.0 & -0.21 & core-jet \\
$1430+412^{*}$ & 1.970 & nHiBAL & 343 & 261.7 & 0.76 & complex \\
$1526+533^{*}$ & 2.822 & HiBAL & 1701 & 182.6 & 0.71 & core-jet/double \\
\hline
\end{tabular}

Table 1: (2) based on SDSS; (3)-(4) taken from [12]; (5) taken from FIRST; (6) $S \propto v^{-\alpha}$, based on FIRST and GB6; (7) radio morphology based on our observations and VIPS images [屯]; $\left(^{*}\right)$ also with EVN observations at $1.6 \mathrm{GHz}$.

region. The result of the SED modeling of $1045+352$ indicates the X-ray emission we observe from $1045+352$ can be mostly due to X-ray emission from the relativistic jet, while the X-ray emission from the corona is absorbed in a large part.

We have performed sensitive high-resolution radio observations of the BAL quasar 1045+352 using the EVN+MERLIN at $5 \mathrm{GHz}$ [7]. The radio morphology of $1045+352$ is dominated by the strong radio jet resolved into many sub-components and changing the orientation during propagation in the central regions of the host galaxy. The presence of the dense environment and material outflows can have a significant impact on the radio jet morphology of 1045+352. We concluded that the most probable interpretation of the observed radio structure of $1045+352$ is the ongoing process of the jet precession due to internal instabilities within the flow and/or jet interaction with a dense, inhomogeneous interstellar medium.

\subsection{Radio observations of a new sample of BAL quasars - preliminary results}

We have prepared a project of multi-frequency radio observations of a new sample of compact radio-loud BAL quasars. The main goal of this project was to study the origin of BALs, jets evolution and probable jet-cloud interactions, by analysis of the BAL QSOs radio morphology. The high resolution radio observations allow to resolve their compact structures and to estimate the orientation of the jet axis what may be a critical parameter in the case of the BAL origin scenarios.

Using the final release of FIRST survey [14] we looked for unresolved, isolated sources i.e. 

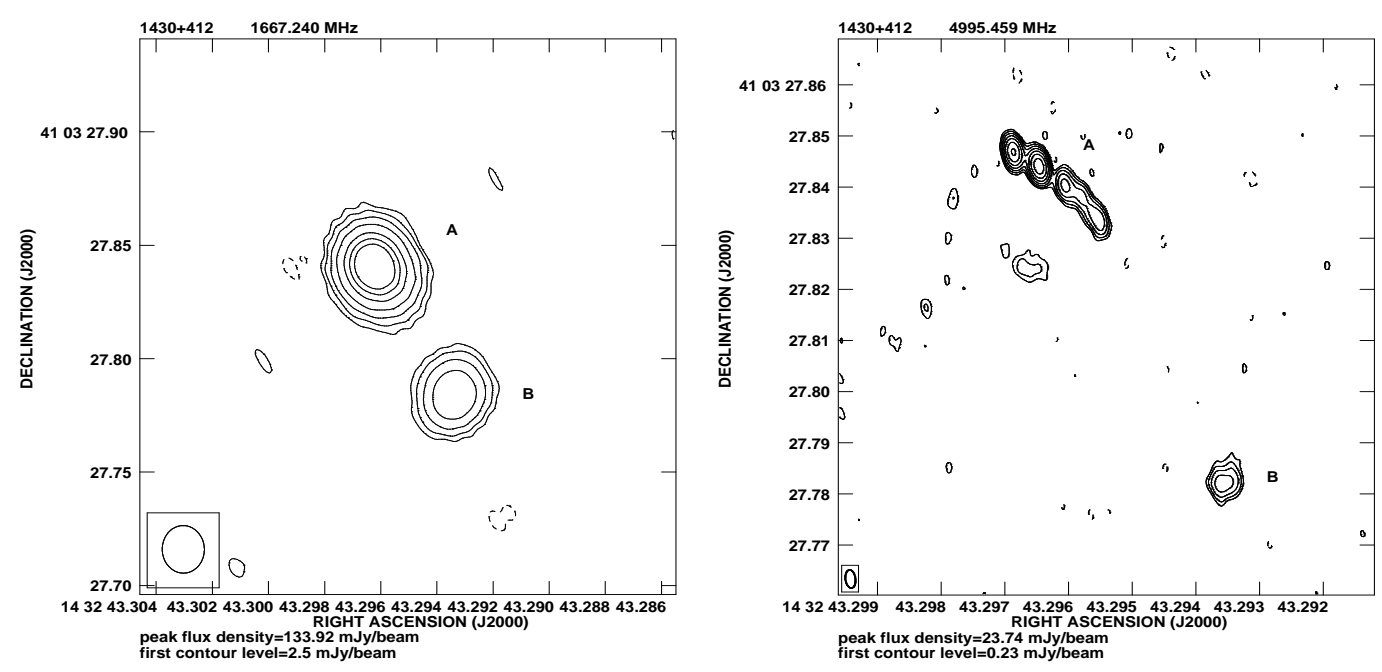

Figure 1: The EVN L-band (left) and VLBA C-band (right) maps of the BAL quasar 1430+412. The positive contour levels increase by a factor of 2 . The Gaussian restoring beams (20.1 mas $\times 16.9$ mas for L-band and 3.6 mas $\times 1.7$ mas for C-band) are shown in the bottom-left corners.

more compact than FIRST beam (5."4) and surrounded by an empty field (we adopted 1' as a radius of that field). We set $150 \mathrm{mJy}$ as the lower flux density limit for the objects at $1.4 \mathrm{GHz}$. We did not put any limits in the case of spectral index, because both GPS and CSS sources can be compact BAL quasars. The next step in our project was a cross-identification of the selected FIRST objects with the optically selected BAL quasars from A Catalog of Broad Absorption Line Quasars from the Sloan Digital Sky Survey Third Data Release [12]. An automated algorithm was used to match the FIRST coordinates with the SDSS position in a radius of $10^{\prime \prime}$. Finally, our sample consists of 16 sources (Table 1 ).

We have observed the sample with VLBA at 5 and $8.4 \mathrm{GHz}$ with polarization detection on 27 \& 30 Aug 2008, 6 \& 7 Sep 2008. The next part of survey was undertaken using EVN at $1.6 \mathrm{GHz}$ and the observations were performed for 10 out of 16 target sources on 27 Oct 2008. However, we have noticed that there is an overlap between our sample of compact BAL quasars and recently published VIPS (VLBA Imaging and Polarimetry Survey) objects [ $₫$ ]. The authors obtained high dynamic range radio maps and polarization information for 5 of our sources at $5 \mathrm{GHz}$. We have continued the observations of our sample in a similar observational strategy to get comparable quality of the radio images for 11 target sources at $5 \mathrm{GHz}$ and 16 objects at $8.4 \mathrm{GHz}$. The target source scan was interleaved with a scan on a phase reference source and the total cycle time (target and phase-reference) was 8 minutes including telescope drive times, with 6 minutes actually on the target source per cycle. The whole data reduction process was carried out using standard AIPS procedures. For the target source, the corresponding phase-reference source was mapped and the phase errors so determined were applied to the target source, which were then mapped using a few cycles of phase self-calibration and imaging. IMAGR was used to produce the final radio images.

All target sources were detected, but they were finally resolved during observations at higher frequencies: 5 and $8.4 \mathrm{GHz}$ which means they are generally very compact sources. Some of them show very complex morphology (Fig. 1 as an example) with probably fading components sug- 
gesting they can be manifestation of the previous activity. This can be explained as caused by the jet-ISM interactions or precession of the jet axis (see also work on the CSS BAL quasar 1045+352 by [四). Most of the observed sources revealed core-jet structures and show fainter emission from the jet compared to the core. This morphology suggest intermediate orientation between polar and equatorial geometries and support the orientation scenario. For some sources the observed flux densities of compact structures account only up to $25 \%$ of total flux density at $5 \mathrm{GHz}$ (on the contrary to $\sim 80 \%$ in the case of sources from [9]). This may suggest that there are low brightness extended structures in our new selected radio-load BALQSOs and sources may be older and bigger than GPS/CSS objects.

It should be noted here, that there are two important observational biases in our sample. First, the selection criteria caused that all sources from the new sample are HiBALs with absorption index $\mathrm{AI}>0$ and balnicity index $\mathrm{BI}=0$. Second, because of the SDSS properties the automated algorithms used by [12] could identify HiBALs via CIV from redshift range $1.7 \leq z \leq 4.38$, therefore the selected sources are probably the most luminous radio-loud BALQSOs. So it is probable that we are probing a special group of radio-loud BAL quasars.

A detailed discussion about the radio properties of the new sample and analysis of their spectral features will be presented in a forthcoming paper.

\section{Acknowledgement}

MERLIN is a UK National Facility operated by the University of Manchester on behalf of STFC. The European VLBI Network is a joint facility of European, Chinese, South African, and other radio astronomy institutes funded by their national research councils. This work was supported by the Polish Ministry of Science and Higher Education under grant N N203 303635.

\section{References}

[1] Becker, R. H., White, R. L., Gregg, M. D. et al., Properties of Radio-selected Broad Absorption Line Quasars from the First Bright Quasar Survey, 2000, ApJ, 538, 72

[2] Ganguly, R., Brotherton, M. S., Cales, S., et al., Outflows and the Physical Properties of Quasars, 2007, ApJ, 665, 990

[3] Elvis, M., A Structure for Quasars, 2000, ApJ, 545, 63

[4] Helmboldt, J. F., Taylor, G. B., Tremblay, S., et al., The VLBA Imaging and Polarimetry Survey at 5 GHz, 2007, ApJ, 658, 203

[5] Jiang, L., Fan, X., Ivezić, Ž., et al., The Radio-Loud Fraction of Quasars is a Strong Function of Redshift and Optical Luminosity, 2007, ApJ, 656, 680

[6] Kunert-Bajraszewska, M., Siemiginowska, A., Katarzyński, K., Janiuk, A., X-rays from a Radio-Loud Compact Broad Absorption Line Quasar 1045+352 and the Nature of Outflows in Radio-Loud Broad Absorption Line Quasars, 2009, ApJ, 705, 1356

[7] Kunert-Bajraszewska, M., Janiuk, A., Gawroński, M. P., Siemiginowska, A., Origin of the Complex Radio Structure in BAL QSO 1045+352, 2010, ApJ, 718, 1345

[8] Montenegro-Montes, F. M., Mack, K.-H., Benn, C. R., et al., Are radio-loud Broad Absorption Line Quasars young sources? , 2009, AN, 330, 157

[9] Liu, Y., Jiang, D. R., Wang, T. G., Xie, F.G., The compact structure of radio-loud broad absorption line quasars, 2008, MNRAS, 391, 246 
[10] Murray, N., Chiang, J., Grossman, S. A., Voit, G. M., Accretion Disk Winds from Active Galactic Nuclei, 1995, APJ, 451, 498

[11] Proga, D. \& Kallman, T. R., Dynamics of Line-driven Disk Winds in Active Galactic Nuclei. II. Effects of Disk Radiation, 2004, ApJ, 616, 688

[12] Trump, J. R., Hall, P. B., Reichard, T. A., et al., A Catalog of Broad Absorption Line Quasars from the Sloan Digital Sky Survey Third Data Release, 2006, ApJS, 165, 1

[13] Weymann, R. J., Morris, S. L., Foltz, C. B., Hewett, P. C., Comparisons of the emission-line and continuum properties of broad absorption line and normal quasi-stellar objects, 1991, ApJ, 373, 23

[14] White, R. L., Becker, R. H., Helfand, D. J., Gregg, M. D., A Catalog of 1.4 GHz Radio Sources from the FIRST Survey, 1997, ApJ, 475, 479

[15] Willott, C. J., Rawlings, S., Archibald, E. N., Dunlop, J. S., A submillimetre difference between radio galaxies and radio quasars: evidence for quasar-heated dust and synchronized submillimetre and radio source activity, 2002, MNRAS, 331, 435

[16] Zhou, H., Wang, T., Wang, H., et al., Polar Outflows in Six Broad Absorption Line Quasars, 2006, ApJ, 639, 716 